\title{
An algorithm for UWB radar-based human detection
}

\author{
SangHyun Chang ${ }^{1}$, Naoki Mitsumoto ${ }^{2}$, Joel W. Burdick ${ }^{1}$ Member, IEEE \\ ${ }^{1}$ Engineering \& Applied Science, California Institute of Technology, MC 104-44, Pasadena, CA 91125, USA \\ ${ }^{2}$ Research laboratories, DENSO Corporation, Aichi, Japan \\ sanghyun@caltech.edu, naoki_mitsumoto@denso.co.jp, jwb@ robotics.caltech.edu
}

\begin{abstract}
This paper presents an algorithm for human presence detection in urban environments using an ultra-wide-band (UWB) impulse-based mono-static radar. A specular multi-path model (SMPM) is used to characterize human body scattered UWB waveforms. The SMPM parameters are used within a classical likelihood ratio detector framework to detect the presence of humans via gait, with the aid of a multi-target tracking technique (MTT). Experimental results on a simple human gait detection problem in an outdoor urban environment are presented to illustrate and validate the approach.
\end{abstract}

Index Terms - UWB radar, impulse radar, human detection, multi-target tracking

\section{INTRODUCTION}

This paper introduces an algorithm for detecting the presence of walking humans in an urban environment populated by other moving objects such as cars and trucks. Our particular motivation is the problem of providing pedestrian safety in the presence of moving vehicles. More generally, the problem of detecting and localizing human presence has been a widely studied problem due to its potential military, safety, security, and entertainment applications. A number of technologies can be used to detect human signatures. Computer vision has limited ability to detect humans in poor visibility conditions (e.g., at night, haze, fog, rain, and smoke, etc.). Similarly, the performance of infrared detectors varies with the ambient temperature conditions. Human LADAR signatures are often not highly discriminable from other moving clutter, and LADAR performance is degraded in dusty and foggy conditions. UWB radar can provide a complementary human detection technology whose performance is less degraded in the poor visibility conditions that plague other sensing technologies.

Human detection using RF, microwave, and mm-wave radar has been previously studied in controlled environments [1], [2], [3], [4], [5], [6]. However, the problem of human detection in real complex environment has been less well addressed. In this paper, we use UWB human scattering properties and human biometric information to develop a UWB monostatic radar-based human detection algorithm. Compared with narrowband radar, UWB radar has many potential advantages for detecting human presence. The target's frequency response over the radar signal's wide bandwidth may provide useful detection and discrimination information. The fine time resolution enabled by wide bandwidth signals not only enables highresolution ranging and localization [7], [8], but also allows

The authors greatly appreciate the financial support of this work provided by the Denso Corporation, Aichi, Japan.
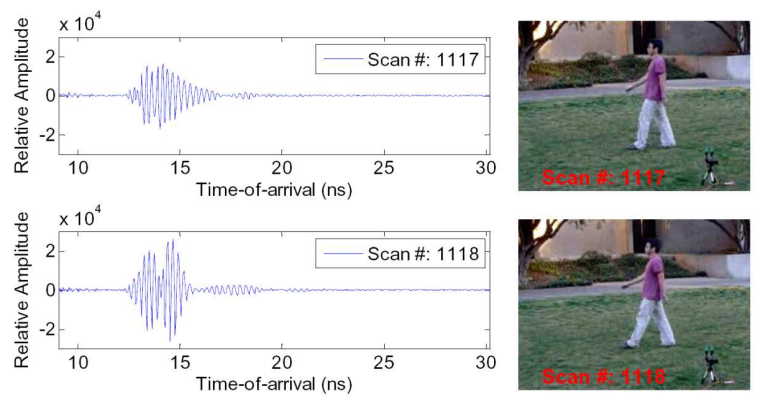

Fig. 1. Scattered UWB waveforms (after antenna coupling removal) and synchronized video image of walking human within the vicinity of a UWB radar.

for highly resolved decomposition of the complex multi-path scatters from the human body. As an example of UWB radar's sensitivity to human body posture, consider Figure 1 which shows video images and returned UWB waveforms of a walking human in an open field, as recorded by synchronized and collocated video camera and monostatic UWB radar. While it is difficult to notice much change in the human's posture across the 0.0326 seconds between frames, note the corresponding scattered UWB waveforms are noticeably different. We use UWB radar's high sensitivity to dynamic posture evolution as the basis for the proposed detection algorithm.

Section II describes a simple UWB signal model, and reviews the CLEAN algorithm for multipath deconvolution. Section III briefly reviews a moving target indication (MTI) approach that complements the signal model of Section II. Section IV models the target-returned UWB radar signal, while Section V describes the basic detection for detecting human bodies undergoing typical gait motions, as well as a multi-target tracking technique (MTT). Experimental results obtained in an urban environment are presented in Section VI.

\section{Specular Multipath Model for UWB Signal}

An effective detection strategy requires a model of UWB radar waveform propagation and interaction with the human body. A perfectly reflecting target, e.g. a metal plate with an infinite area, returns the impinging UWB electromagnetic wave along a single-path. However, for targets characterized by complex shapes in the scale of the spatial extent of the transmitted UWB signal pulse width, e.g. the human body, the returned UWB radar signal consists of multipath components [9], as the impinging UWB electromagnetic wave scatters independently from different human body parts at different times with various amplitudes (depending on the distance to 
the body part and the size, shape, and composition of the scattering part). Each of these different scattering pathways can be considered as multipath components of the returned UWB radar signal. In addition, time variation of the scattered UWB waveform can be introduced by the movement of scatterers or the movement of the transmitter or receiver [10].

In the domain of UWB radio communication channel modeling, Tsao, et al., proposed the time-evolving UWB multipath channel model [11]:

$$
h(t, \tau)=\sum_{j=1}^{L} a_{j}(t) p_{j}\left(\tau-n_{j}(t)\right)
$$

where $\tau$ is the delay, and $t$ is the elapsed time. The channel model $h(t, \tau)$ is considered to be the superposition of the $L$ strongest scattering paths, where each path represents the response at time $t$ to an impulse transmitted at time $t-\tau$. Path $j$ is specified by the path time-of-arrival (TOA) $n_{j}(t)$, the path amplitude $a_{j}(t)$, and the path waveform $p_{j}(\cdot)$. It is impractical to apply the model in Equation (1) to mono-static radar sensing in the presence of a real time processing constraints. To make the channel model tractable, Equation (1) can be approximated by the specular multipath channel model [12]:

$$
h(t, \tau) \approx \sum_{j=1}^{L} a_{j}(t) \delta\left(\tau-n_{j}(t)\right),
$$

where $\delta(\cdot)$ is the Dirac delta function. This simplification allows for real-time processing without compromising UWB radar's high time-resolution capability ${ }^{1}$,

Hence, the radar signal returned by a mono-static UWB radar, $w(t)$, can be represented by a specular multipath model:

$$
w(t) \approx \sum_{j=1}^{L} a_{j}(t) p\left(t-n_{j}(t)\right)
$$

where $w(t)=\int_{-\infty}^{\infty} h(t, \tau) p(t-\tau) d \tau$ as in [14], and $p(\cdot)$ is an elementary waveform shape (e.g., the received waveform in free space). Note that the path amplitude $a_{j}(t)$ represents the product of attenuation factors due to the transmnitter and receiver antenna patterns, as well as the scatterer configuration. The path TOA $n_{j}(t)$ represents propagation delay at time $t$ from the transmitter to the receiver on path $j$. Assuming that multiple scattering events do not occur on the path, we can convert between (round-trip) propagation delay and scatterer range, as the range $R_{j}(t)$ of the scatterer on path $j$ is $R_{j}(t)=$ $\left[n_{j}(t) \cdot c\right] / 2$, where $c$ is the speed of light $^{2}$. For example, when a transmitted UWB signal sounds the channel every $T_{s}$ seconds, the $\mathrm{m}^{t h}$ scan can be represented as the waveform $w(t)$ recorded over a sweeping interval $t \in\left[t_{0}+(m-1) T_{s}, t_{\max }+\right.$

\footnotetext{
${ }^{1}$ The specular multipath model is a quite simplified model, since wave reflection from and wave penetration through target material are in reality frequency sensitive. Therefore the waveform is filtered in some way. Thus, the returned waveform may vary over each of the path components [13].

${ }^{2}$ We refer to propagation delay and range interchangeably in the paper.
}

$\left.(m-1) T_{s}\right]$, which corresponds to a range of $r \in\left[r_{0}, r_{\max }\right]=$ $\left[c t_{0} / 2, c t_{\max } / 2\right]^{3}$.

To estimate the channel parameters (the amplitude and TOA of each multipath component) in Equation (2) for a radar scan $w(t)$ over a recording interval, a given path waveform shape template, $v(t)$, is used to deconvolve the path components from $w(t)$ using a variant of the CLEAN algorithm. The CLEAN algorithm, firstly introduced in [15] and well established in the radio astronomy and microwave communities, has been applied to impulsive UWB measurements in [13], [16]. The algorithm searches the received waveform iteratively with the template to find the maximum correlations, which correspond to the various channel components. The iteration stopping criterion is based on a minimum threshold on the peak correlation. However, the the channel parameters estimated by the conventional CLEAN algorithm may provide an unsuitable representation for our radar application, since for a fixed stopping threshhold, the number of detected reflection paths from a target is a function of target range (due to free space loss, the path amplitude decreases inversely proportional to the square of target range). Therefore, we propose a rangeadaptive threshold, which is inversely proportional to the square of each detected path's range, to provide a consistent target representation in target range variation ${ }^{4}$.

\section{CLEAN Algorithm Summary}

1. Input : Waveform shape template $v(t)$; and detection threshold $T_{\text {clean }}$ normalized at 1 meter.

2. Initialize : Form initial residual waveform $d_{0}(t)=w(t)$ for a scan. Set iteration counter $i=0$.

3. Signal Detection : Compute cross-correlation $r_{v d}(\tau)$ between $v(t)$ and $d_{i}(t)$; the time-index associated to the maximum magnitude of $r_{v d}(\tau)$ is the $i^{t h}$ estimated TOA:

$$
\hat{n}_{i}(t)=\arg \max _{\tau}\left|r_{v d}(\tau)\right| \text {. }
$$

The cross-correlation at $\hat{n}_{i}(t)$ is the $i^{\text {th }}$ estimated amplitude:

$$
\hat{a}_{i}(t)=r_{v d}\left(\hat{n}_{i}(t)\right) \text {. }
$$

If the path magnitude is below the threshold at the TOA, STOP.

4. Increment the iteration counter $: i \leftarrow i+1$.

5. Residual waveform update:

6. Iterate : Go to step 3 .

\section{Moving TARget IndicAtion AND SEgmentation}

Since UWB radar can sense both stationary and moving objects, all scatters obtained from a complex test environment must be analyzed for potential human target candidates. To reduce the high computational cost associated to such analysis, a moving target indication (MTI) system [17] rejects highly human-unlike stationary clutter. Let the monostatic radar's range $\left[r_{0}, r_{\max }\right]$ be divided in $N_{\text {bin }}$ bins of length $D_{m} \mathrm{~m}$.

\footnotetext{
${ }^{3}$ The channel coherence time is assumed to be much larger than the recording time length, $t_{\max }-t_{0}$, so that the path amplitude $a_{j}(t)$ and the path TOA $n_{j}(t)$ are constant during the sweeping time interval.

${ }^{4}$ To avoid high false alarm rate on path detection, especially at far range, the effective noise level should be below the range-adaptive threshold level.
} 

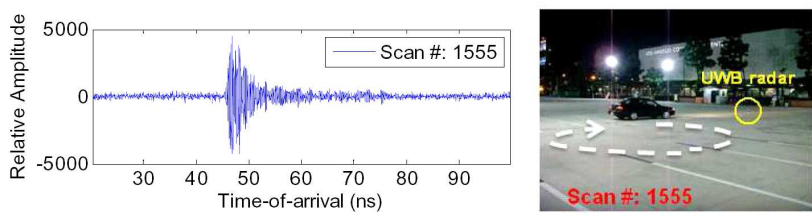

Fig. 2. Scattered UWB waveform (after antenna coupling removal) and synchronized video image of a car moving within the vicinity of a UWB radar.

The UWB radar scans are repeated every $T_{s}$ seconds. The degree of the environment's stationarity in the $k^{\text {th }}$ range bin is measured by the scattered waveform variance in that range bin over $t_{m}$ seconds (where $t_{m}$ is some integer multiple of $T_{s}$ ). If the variance is below a threshold $T_{m}$, then the signal in the $k^{\text {th }}$ range bin is considered stationary, and the mean of the signal over $t_{m}$ seconds is canceled in subsequent scans as a stationary clutter component. Note that the MTI system also typically rejects the direct coupling component between transmitting and receiving antennas.

When multiple moving objects exist within the radar's range, the return signal must be segmented in time to isolate the scatters associated with individual moving objects, and subsequently analyzed by the human detector of Section V. Compared with Doppler radar, UWB radar can effectively resolve multiple targets by range gating, even without employing an array antenna, since a UWB signal has much wider RF Gabor (RMS) bandwidth than a Doppler radar signal [8]. To exploit UWB radar's high range-resolution capability, the segmentation process is applied on the CLEAN algorithm output. Thus, for example, resolved path components within $D_{s g}$ m apart (or $D_{s g} / 2 c$ seconds of TOA apart) can be regarded as scattered from the same object, so that the multipath components are clustered to be associated with the same objects. See [18] for a more sophisticated probabilistic segmentation algorithm.

\section{Model of Target-Returned UWB Radar Signal}

The specular multipath model Equation (3) is a computationally useful signal representation that reduces UWB waveform representation to 2 dimensions (path amplitude and TOA). To differentiate human scatter from non-human scatter, discriminatory target features are required. Three features are proposed for discriminating human and non-human scatter under the specular multipath model: (1) the path's maximum magnitude, which is relevant to target composition and crosssection size; (2) the RMS delay spread of multipath delay profile (or the RMS range spread), which is relevant to target size over the range dimension; and (3) the velocity of target.

To establish a model for the features of human scatters, we constructed a database of UWB radar scans obtained while a human randomly walked in an open field within the vicinity of a stationary monostatic UWB radar as in Figure 1. An analogous database was constructed from UWB radar scans obtained from a car moving in spacious empty parking lot as in Figure 2, since automobiles are apt to be the most abundant moving objects other than humans in urban outdoor

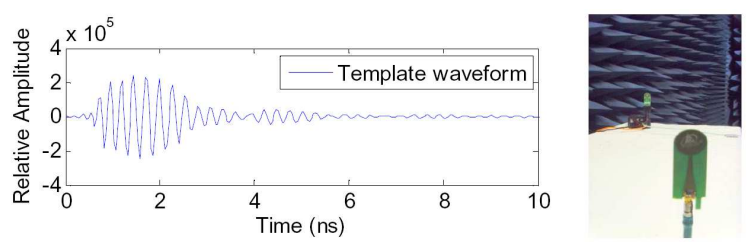

Fig. 3. Measured template waveform (left) and measurement setup (right)

environment ${ }^{5}$. On each of databases, the MTI system response of UWB radar scans (Section III) are applied to the CLEAN algorithm (Section II) in order to extract the discriminatory target features based on the specular multipath model. Figure 3 shows the template waveform shape measured in approximate free space ${ }^{6}$.

The empirical distributions of the maximum magnitude feature are shown in Figure 4(a) as histograms. Also included are a Gaussian curve-fit to the human distribution, and a mixture of two Gaussian distributions for car scatters. Since both human and automobile usually generate multiple scatter components, the maximum amplitude of any path associated with the target is used to construct the histograms. Moreover, since the scattered path amplitude is inversely proportional to the square of the scatterer's range, we normalize the path amplitudes to a common 1 meter range reference. From Equation (3), the path's maximum magnitude can be represented as

$$
a_{\max }=\max _{j \in \Omega}\left|a_{j} R_{j}^{2}\right|
$$

where $\Omega$ is the set of path indices associated with the target, and $R_{j}^{2}=\left(c n_{j} / 2\right)^{2}$ is normalization factor. The maximum return signal magnitude $(\sim 135 \mathrm{~dB}$ in Figure $4(\mathrm{a}))$ occurs when the radar faces either of the car's sides, which happens when the car's moving direction is perpendicular to wave incident/returning direction.

Based on the human and car scattering databases, empirical distribution histograms of the human and car RMS range spreads are shown in Figure 4(b) with their Gaussian curvefits. From Equation (3), the first moment of the power range profile is defined as a function of normalized path amplitude by $\bar{R}=\frac{\sum_{j \in \Omega} R_{j}\left(a_{j} R_{j}^{2}\right)^{2}}{\sum_{j \in \Omega}\left(a_{j} R_{j}^{2}\right)^{2}}$. Then, the RMS range spread $R_{r m s}$ can be calculated as

$$
R_{r m s}=\left[\frac{\sum_{j \in \Omega}\left(R_{j}-\bar{R}\right)^{2}\left(a_{j} R_{j}^{2}\right)^{2}}{\sum_{j \in \Omega}\left(a_{j} R_{j}^{2}\right)^{2}}\right]^{\frac{1}{2}} .
$$

Figure 4(c) shows constructive distributions of the human and car velocities, where presumed human velocity has a cutoff at $10 \mathrm{~m} / \mathrm{s}$. The extraction of velocity feature $v$ in

\footnotetext{
${ }^{5}$ Measurements were conducted using a Time Domain PulsOn 210 monostatic radar (TDR) with a waveform sampling period of $\sim 41.33 \mathrm{ps}$. For the human and car databases, the number of scans acquired was 1161 and 1788 with scanning frequency of $30.7 \mathrm{scans} / \mathrm{sec}$ and $9.7 \mathrm{scans} / \mathrm{sec}$, respectively. For car database, a black Toyota Corolla rotated circularly aside by TDR to measure radar responses for whole azimuth angles, where the rotating axis was about $9 \mathrm{~m}$ far from TDR with the rotatiing radius around $5 \mathrm{~m}$.

${ }^{6}$ The template waveform was measured by using the TDR on a styrofoam supporting structure in an RF anechoic chamber. The receiving antenna was 1 $\mathrm{m}$ far from the transmitting antenna, separated by using an extra SMA cable.
} 


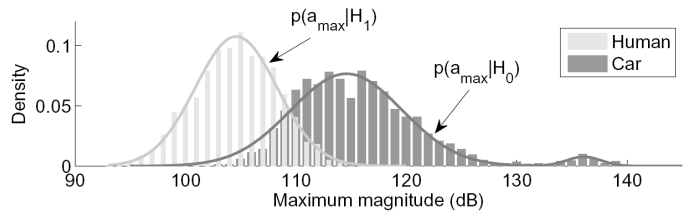

(a) Path maximum magnitude

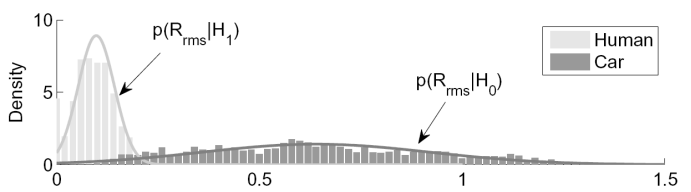

(b) The RMS range spread

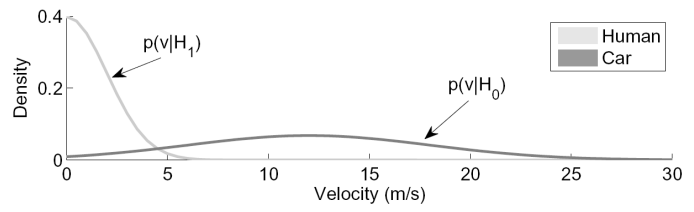

(c) The target velocity (presumed)

Fig. 4. Empirical distributions with curve-fit and constructive distributions of three features for human scatters and car scatters.

a real complex test environment requires not only a stable target tracking system (e.g., a Kalman filter), but also a data association procedure to associated target returns to tracks across radar recording intervals ${ }^{7}$. We employed a multi-target tracking (MTT) technique, whose details are discussed below.

\section{UWB RADAR-BASEd Human Detector Framework}

A human detector framework is proposed in Figure 5, describing a single algorithm cycle for each updated radar scan. Firstly, the moving target indication (MTI) system of Section III is applied to each incoming radar scan. Secondly, the CLEAN algorithm in Section II is applied to the MTI response of radar scan to obtain estimated TOAs and amplitudes of the decomposed multipath components. Thirdly, the segmentation procedure described in Section III is applied to output of the CLEAN algorithm. Fourthly, each segment is be associated to segments from previous recording intervals, thus tracking the scatters from moving targets by exploiting a multi-target tracking (MTT) technique. Fifthly, for each track, the features of maximum magnitude, RMS range spread, and velocity are estimated. Finally, a hypothesis testing process determines whether the tested track is interpreted/detected as a human or not. Then, a detector combines the result of the detection process on all segments to declare human presence if there exists at least one segment where a human is detected. Otherwise, no human presence is declared. The following subsection will describe a multi-target tracking technique in detail, as well as the detection process.

\section{A. Multi-target Tracking (MTT)}

For multiple human tracking via UWB radar, the Kalman Filter combined with the expectation maximization (EMKF)

\footnotetext{
${ }^{7}$ By the velocity feature, we mean the radial velocity due to the limitation of TDR with single transmitting and single receiving antennas.
}

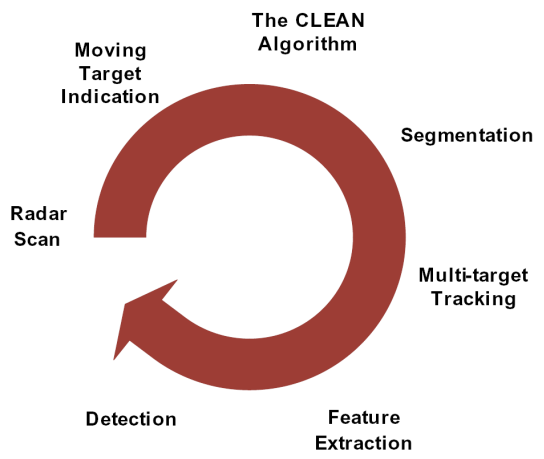

Fig. 5. An algorithm cycle of human detector framework.

algorithm has been proposed for fixed and known number of target to tracking in [18]. However, In complex environment, there may be multiple targets to track, which may include the case when some targets go in and/or out an observation volume to have a variable number of target. Therefore, it is necessary to develop a multi-target tracking (MTT) solution which allows for changing numbers of targets across radar scans, false measurements (clutter), and missed detections (temporary occlusions).

An abundance of established MTT literature exists, primarily intended for military and, more recently, computer vision applications [20], [21], [19]. However, the key differentiator of MTT for UWB radar recording versus traditional applications is the multitude of observations (multipath scattering) per target in each scan, due to the short spatial extent of the transmitted UWB signal pulse width. Wolf recently developed Multi-Hypothesis Cluster Tracking (MHCT) algorithm in the application of spike sorting in extracellular neural recordings [22]. Based on the similar multitude nature of observations, a variant of Wolf's MHCT is employed to UWB radar application, which may provide the velocity feature.

\section{B. Detection}

The detection process on each segment can be viewed as a binary hypothesis test over the segment's observation $\Theta=\left\{a_{\max }, R_{r m s}, v\right\}$ of the following null hypothesis, $\mathcal{H}_{0}$, and the alternative hypothesis, $\mathcal{H}_{1}$ :

$$
\begin{aligned}
& \mathcal{H}_{0} \text { : no human is present } \\
& \mathcal{H}_{1} \text { : human is present. }
\end{aligned}
$$

The hypotheses are evaluated using a likelihood ratio test (LRT) $\Lambda(\Theta)$ :

$$
\Lambda(\Theta)=\frac{\mathcal{L}\left(\Theta \mid \mathcal{H}_{1}\right)}{\mathcal{L}\left(\Theta \mid \mathcal{H}_{0}\right)}=\frac{p\left(\Theta \mid \mathcal{H}_{1}\right)}{p\left(\Theta \mid \mathcal{H}_{0}\right)}
$$

where the likelihood function $\mathcal{L}\left(\Theta \mid \mathcal{H}_{i}\right)$ given segment observation $\Theta=\left\{a_{\max }, R_{r m s}, v\right\}$ under the hypothesis $\mathcal{H}_{i}$ is defined by the conditional probability distribution $p\left(\Theta \mid \mathcal{H}_{i}\right)$ of three features under the hypothesis $\mathcal{H}_{i}$, for $i=0,1$. Provided that $a_{\max }, R_{r m s}$, and $v$ are all independent, the LRT has the form:

$$
\Lambda(\Theta)=\frac{p\left(a_{\max } \mid \mathcal{H}_{1}\right) p\left(R_{\text {rms }} \mid \mathcal{H}_{1}\right) p\left(v \mid \mathcal{H}_{1}\right)}{p\left(a_{\max } \mid \mathcal{H}_{0}\right) p\left(R_{\text {rms }} \mid \mathcal{H}_{0}\right) p\left(v \mid \mathcal{H}_{0}\right)} \underset{\mathcal{H}_{1}}{\stackrel{\mathcal{H}_{0}}{\lessgtr}} T_{D}
$$


where the six conditional probability distributions ${ }^{8}$ are plotted in Figure 4, and the detection threshold $T_{D}$ determines the performance of the detection process.

The basic detector combines the results of the LRT only for maximum amplitude $a_{\max }$ and RMS range spread $R_{r m s}$ features over each segment to evaluate the probability of human presence in each radar scan. While this basic detector can be processed quickly with low latency, it does not utilize mutual information or dynamics across successive scans. To integrate information over time, we use a voting method that calculates number of times that human presence is detected over the last $N_{v}$ basic detector cycles. Human presence is declared when greater than $50 \%$ of the $N_{v}$ scans vote positively for human presence. The MHCT detector combines the results of the LRT in Equation (4), adding the velocity $v$ feature to the basic detector. Also, the voting method can be applied to the MHCT detector output, which may improve the accuracy of track confirmation and deletion process. Experimental receiver operating characteristics (ROCs) obtained by applying the basic and MHCT detectors to a UWB monostatic radar operating in an outdoor urban environment are presented next.

\section{EXPERIMENTAL RESULTS}

A sequence of UWB radar scans (test parameters are summarized in Table I) were measured using a Time Domain PulsOn 210 monostatic UWB radar placed at 0.5 meter height above the curbside of California Blvd. on the California Institute of Technology campus. Figure 6(a) shows 3000 unprocessed scans (each column represents the magnitude of a single scanned waveform over the sensing range, where the waveform magnitude is scaled from 0 in black to 3000 in white. Magnitudes over 3000 are clipped to 3000). During the $\sim 10$ minute recording time, several humans and cars moved in and out of radar range. The radar's range also included fixed objects (e.g. metal poles, a wall, and several trees). Some moving object trajectories can be seen in Figure 6(a), while the horizontal patterns represent stationary scatterers, as well as direct antenna coupling effects in the near range. Figure 6(b) shows MTI responses ${ }^{9}$ of walking human scatters, the output of the CLEAN algorithm and segmentation steps for the $92^{\text {nd }}$ scan, and the synchronized video image.

The UWB radar-based human detector of Figure 5 was tested on a subset of 2486 scans of the 3000 scans during which the number of humans in the test environment is

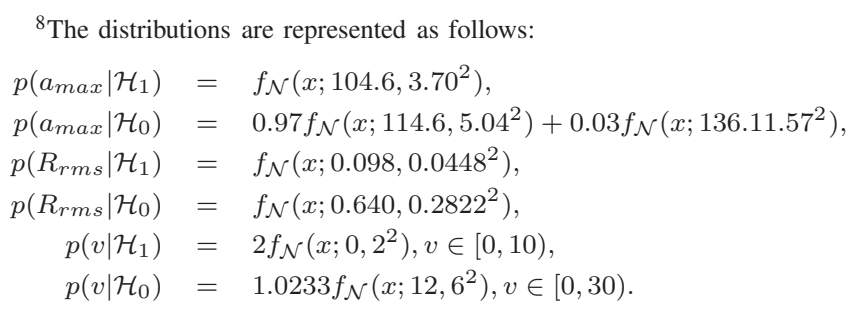

where normal distribution with the mean $m$ and the variance $\sigma^{2}$ is denoted by $f_{\mathcal{N}}\left(x ; m, \sigma^{2}\right)$ on the domain $x \in(-\infty, \infty)$.

${ }^{9}$ Direct antenna coupling effects over the range $0.3014 \mathrm{~m}$ to $2.155 \mathrm{~m}$ were also removed in this step.

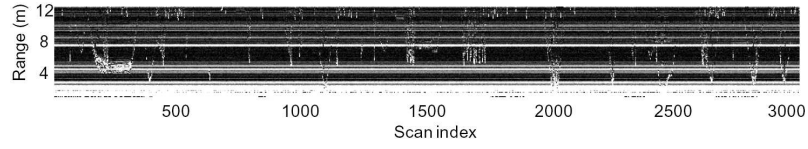

(a) Representation of 3000 unprocessed scans.
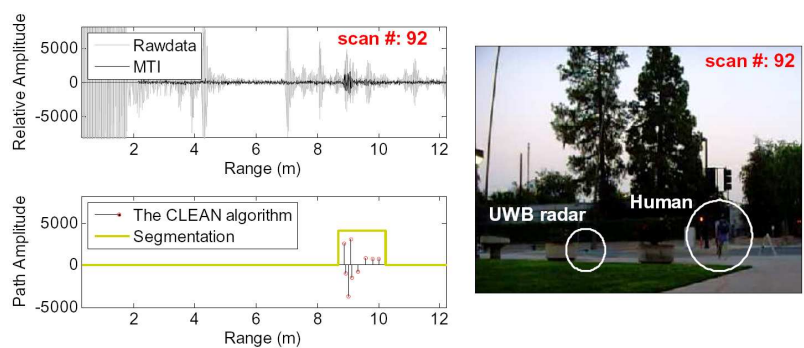

(b) Signal processing for scan 92, with synchronized video image.

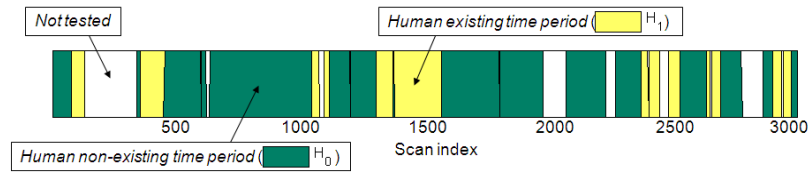

(c) Radar range description over the tested recording interval.

Fig. 6. Experimental results in an outdoor urban environment.

controlled to be always 0 or 1 . Figure 6(c) shows that 1778 scans without human presence and 708 scans including human presence are tested by the basic and MHCT detectors of Equation (4). We evaluated the basic detector with threshold values $T_{D}=1,1.5,2, \cdots, 5.5$ and voting intervals including $N_{v}=1,2,4, \cdots, 14$ scans. Also the MHCT detector was evaluated with threshold values $T_{D}=2,2.5,3,3.5,4$ and voting intervals of $N_{v}=1,2,4,6$ scans. The basic detector's receiver operating characteristic (ROC) under these different conditions is shown in Figure 7(a). Clearly, the basic detector with the voting method performs better than the basic detector without the voting method $\left(N_{v}=1\right)$, and the detection performance improves with increasing $N_{v}$, where the performance saturates at $N_{v}=12$. The basic detector with $N_{v}=14$ and $T_{D}=4$ provides an $80.7 \%$ probability of human presence detection for $5.46 \%$ false alarm rate. The voting method results in the basic detector show that integration of radar information over time significantly improves performance. However, the latency of the basic detector with $N_{v}=12$ is 2.46 seconds, which demonstrates the trade-off between detection performance and latency.

The MHCT detector results show that the additive velocity feature via the MHCT approach significantly improves performance in Figure 7(b), compared to the basic detector for the same voting intervals. Also, the MHCT detector performance with the voting method is improved except when $N_{v}=2$ due to different target confirmation and deletion logics from other voting intervals. The MHCT detector with $N_{v}=6$ and $T_{D}=3$ provides an $82.74 \%$ probability of human presence detection for $1.58 \%$ false alarm rate. Figure 7 (c) shows detection errors of false alarm (Type-I error) and missed detection (Type-II error) types with the corresponding scan index. In analyzing the failed detections, we observed that the majority of the 


\begin{tabular}{|c|c|c|c|}
\hline Radar measurement setup & Algorithm parameters & Kalman filter parameters & MHCT parameters \\
\hline 1TX and 1RX antennas & $D_{m}=0.4 \mathrm{~m}$ & $A=\mid \begin{array}{cc}1 & T_{s} \\
0 & 1\end{array}$ & $K=6$ \\
\hline $\begin{array}{c}\text { Height: } 1 \mathrm{~m} \\
\text { Radar range: } 0.3 \sim 12.2 \mathrm{~m} \\
\text { Number of scans recorded: } 3000 \mathrm{scans} \\
\text { Scanning period: } T_{s}=0.2050 \mathrm{sec} \\
\text { Range sampling resolution: } \Delta R=0.0062 \mathrm{~m} \\
\text { Hardware \& software average: } 2 \& 512\end{array}$ & $\begin{array}{c}N_{\text {bin }}=30 \mathrm{bins} \\
t_{m}=0.82 \mathrm{sec} \\
T_{m}=7 \times 10^{4} \\
D_{m}=0.4 \mathrm{~m} \\
D_{\text {sg }}=0.5 \mathrm{~m} \\
T_{\text {clean }}=5 \times 10^{4}\end{array}$ & $\begin{array}{r}H=\left[\begin{array}{cc}1 & 0\end{array}\right] \\
q=\frac{2 T_{s}}{3 \Delta R} \\
R_{k}=\left(\frac{0.1}{\Delta R}\right)^{2}\end{array}$ & $\begin{array}{c}\lambda_{\nu}=0.01 \\
\lambda_{\phi}=0.0105 \\
\mathrm{P}_{\mathrm{d}, j}=0.3 \\
\mathrm{P}_{\mathrm{d}, \max }=0.98, \mathrm{P}_{\mathrm{d}, \min }=0.3 \\
K_{\text {miss }}=1 \\
N_{D}=5\end{array}$ \\
\hline
\end{tabular}

TABLE I

HUMAN DETECTOR TEST PARAMETERS.

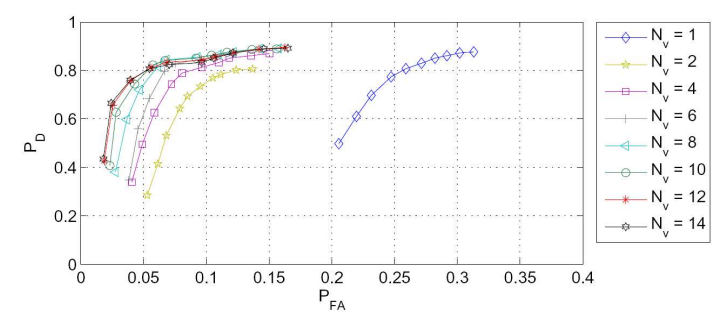

(a) ROC curves of the basic detector.

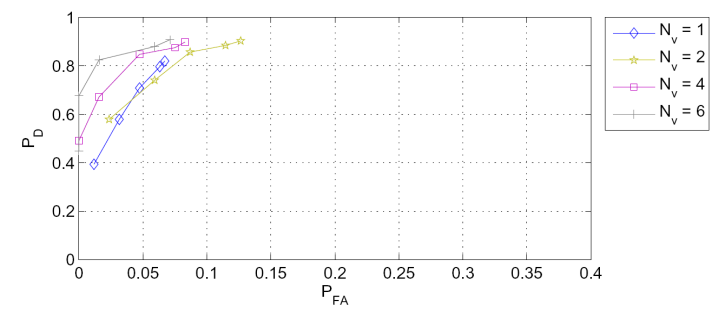

(b) ROC curves of the MHCT detector.

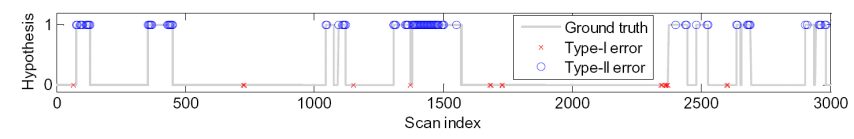

(c) The MHCT detector errors for $N_{v}=14$ and $T_{D}=4$.

Fig. 7. Results of the basic and MHCT detectors.

errors occurred during transitions of hypothesis-i.e., a human was entering or leaving the radar range.

\section{CONCLUSION}

Even with only three features, the UWB radar-based human detector has better than $80 \%$ detection probability with $1.58 \%$ false alarm rate in a realistic outdoor environment. While we only tested the detector in conditions containing fixed objects, moving humans, and moving cars, it is likely that the maximum magnitude, RMS range spread, and velocity features may be useful for discriminating humans from common clutter, such as small animals and bicycles, which might arise in a surveillance application. To improve detection performance, better segmentation techniques or feature aided tracking can be employed. The superior performance with the voting method also suggests that the use of a more dynamic signal model that integrates information across scans, such as a hidden Markov model, should result in improved performance. These ideas are the subject of ongoing work.

\section{REFERENCES}

[1] Z. Zhang, P. O. Pouliquen, A. Waxman, and A. G. Andreou, "Acoustic micro-Doppler radar for human gait imaging," J. Acoustical Soc. America Express Letters, vol. 121, no. 3, pp. 110-113, March 2007.

[2] S. Z. Gurbuz, W. L. Melvin, and D. B. Williams, "Comparison of RadarBased Human Detection Techniques, "Proc. 41 ${ }^{\text {st }}$ Asilomar Conf. on Signals, Systems and Computers, pp. 2199-2203, 2007.

[3] J. L. Geisheimer, E. F. Greneker, and W. S. Marshall, "A high-resolution doppler model of human gait," Proc. of SPIE, vol. 4744, 2002.

[4] P. Van Dorp, and F. C. A. Groen, "Human walking estimation with radar," IEE Proc. on Radar, Sonar and Nav., 150(5):356-365, 2003.

[5] M. Otero, "Application of a continuous wave radar for human gait recognition," Proc. of SPIE, vol. 5809, pp. 538-548, 2005.

[6] N. Yamada, Y. Tanaka, and K. Nishikawa, "Radar Cross Section for Pedestrian in 76GHz Band," Proc. European Microwave Conference, vol. 2, 2005. t and order," April 2002. ET-Docket 98-153.

[7] J. D. Taylor, Introduction to Ultra-Wideband Radar Systems, $1^{\text {st }}$ ed., Boca Ranton, FL: CRC, 1995.

[8] R. A. Scholtz, D. M. Pozar, and W. Namgoong, "Ultra-Wideband Radio, EURASIP J. Applied Signal Processing, No. 3, 2005, pp. 252-272. 5. G. Franceschetti, C.

[9] E. F. Knott, J. F. Shaeffer, and M. T. Tuley, Radar Cross Section, $2^{\text {nd }}$ ed., Artech House, Norwood, MA, 1993.

[10] A. F. Molisch, "Time-variance for UWB wireless channels," IEEE, Tech. Rep. Document IEEE 802.15-02-000-00-003a, 2002.

[11] J. Tsao, D. Porrat, and D. Tse, "Prediction and modeling for the timeevolving ultra-wideband channel," IEEE Journ. Selected Topics in Sig. Proc., vol. 1, no. 3, pp. 340.356, Oct. 2007.

[12] H. Hashemi, "Impulse response modeling of the indoor radio propagation channels," IEEE Trans. Select. Areas Commun., vol. 11, no. 7, Sep. 1993.

[13] R. J.-M. Cramer, R. A. Scholtz, and M. Z. Win, "An evaluation of the ultra-wideband propagation channel," IEEE Trans. Antennas and Prop., vol. 50, pp. 561-570, May 2002.

[14] P. A. Bello, "Characterization of randomly time-variant linear channels," IEEE Trans. Comm., vol. 11, pp. 360-393, 1963.

[15] J. A. Högbom, "Aperture synthesis with a non-regular distribution of interferometer baselines," Astron. and Astrophys. Suppl. Ser., vol. 15, 1974.

[16] S. M. Yano, "Investigating the ultra-wideband indoor wireless channel," in Proc. IEEE VTC Spring Conf., vol. 3, pp. 1200.1204, 2002.

[17] M. I. Skolnik, Radar Handbook, New York: McGraw Hill, 1970.

[18] S. Chang, R. Sharan, M. Wolf, N. Mitsumoto, and J.W. Burdick, "UWB radar-based tracking of humans," to appear in RadarCon, Pasadena, CA, May 4-8, 2009.

[19] G. W. Pulford, "Taxonomy of multiple target tracking methods," IEE Proceedings - Radar, Sonar \& Navigation, 152(5):291 304, 2005.

[20] Y. Bar-Shalom and T. E. Fortmann, Tracking and Data Association, Academic Press Inc., Orlando, FL, 1988.

[21] S. S. Blackman and R. Popoli, Design and Analysis of Modern Tracking Systems, Artech House, Norwood, MA, 1999.

[22] M.T. Wolf, "Target Tracking Using Clustered Measurements, with Applications to Autonomous Brain-Machine Interfaces," Ph.D. thesis, Department of Mechanical Engineering, California Institute of Technology, Pasadena, CA, June 2008. 